\title{
Diagnostics cliniques comme constructions sociales
}

\author{
Volkmar Aderhold
}

\author{
Psychotherapie-Wissenschaft 8 (1) 33-34 2018 \\ www.psychotherapie-wissenschaft.info \\ CC BY-NC-ND \\ DOI: $10.30820 / 8242.06$
}

Mots-clés : Diagnostics psychiatriques, ICD, DSM, comorbidité, décontextualisation, biologisation, Research Domain Criteria $(\mathrm{RDOC})$, diagnostic comme processus basé sur la communication, polyphonie

Les manuels de diagnostics de la décennie passée avaient pour effet ou visaient à une décontextualisation avancée des troubles psychiques, accompagnée d'une biologisation avancée de la psychiatrie, qui au final sert davantage à l'industrie pharmaceutique qu'aux patients. Dans ce contexte, l'ensemble des résultats de la psychiatrie biologique est tout sauf encourageant, notamment également parce que les catégories de diagnostics sont trop peu valides. Le nouveau système du Research Domain Criteria (RDoC) qui émane de sous-systèmes neuronaux fonctionnels du cerveau sain pourrait faire avancer la neurobiologie. Il est impossible de dire si cela va marcher.

Les modifications des catégories de diagnostics et des critères au sein des groupements sont énormes, au-delà des différentes versions des manuels de diagnostics. La tentative de la DSM-5 également de reclasser les diagnostics le démontre à nouveau. Le diagnostic de schizophrénie montre clairement à quel point les tentatives de classification des manuels de diagnostics sont arbitraires. Historiquement, l'intersection par ex. des groupements de symptômes utilisés pour la schizophrénie depuis sa découverte par Bleuler (1911) jusqu'en 1984 se situe à $27 \%$, à savoir plus de $70 \%$ des gens qui sont définis ainsi dans un système ne seraient pas conservés dans un autre système de diagnostic. Pour les personnes concernées, cela a toutefois une importance déterminante sur le plan biographique que cette maladie leur soit ou non diagnostiquée.

Les sous-groupes de la schizophrénie selon Bleuler (1911) - hébéphrène, catatonique, paranoïde et indifférencié - sont aussi justement supprimés par le DSM-5 en raison d'une faible stabilité, fiabilité et d'une faible pertinence pronostique. De la même façon, les symptômes de 1er rang de Kurt Schneider ne sont plus utilisés comme critères. Schneider les avait à l'origine (1938) certes uniquement acceptés sur un plan heuristique, ils ont toutefois été considérés pendant des décennies comme " pathognomoniques ", à savoir suffisants pour un établissement sûr de diagnostic. Au bout de plus de 70 ans, ils n'ont pas été confirmés sur le plan empirique. Un empirisme tardif pour un diagnostic scientifique soi-disant basé sur des preuves! Pour diagnostiquer une schizophrénie, que ce soit dans l'ICD-10 ou dans l'ICD-11 à venir, une période de symptômes de quatre semaines est suffisante, dans le
DSM c'est au contraire six mois. C'est une sévère différence qui continue à exister également après trois révisions entre les groupes d'experts. Cela démontre une nouvelle fois à quel point des décisions sont prises arbitrairement, des décisions qui néanmoins ont des conséquences graves sur de nombreux(ses ) clientes et clients.

La bonne intention et le bon objectif de rendre l'établissement du diagnostic indépendant de l'orientation théorique du diagnosticien sont devenus une antithèse: Avec les théories, les contextes psychosociaux ont également été perdus de vue. Et bien qu'aujourd'hui les diagnostics semblent être posés indépendamment de l'origine, l'établissement du diagnostic génère souvent une biologisation implicite et explicite et " une généticisation » des constructions syndromiques. Les deux présentent des inconvénients. Cette décontextualisation a souvent pour conséquence que le diagnostic génère des suites défavorables ou erronées pour les personnes concernées. Car la biologisation ouvre trop souvent la voie à la pharmacothérapie primaire, voire exclusive. $\mathrm{Ci}$-après, je présente en détail les inconvénients.

Les diagnostics ont souvent un caractère stigmatisant, rarement libératoire. La tentative de réduire l'ampleur de la stigmatisation de " maladies " psychiques par leur biologisation a vraisemblablement échoué. Avec l'expansion sociétale de diagnostics aux modèles explicatifs biologiques inventés, la stigmatisation sociale augmente plus qu'elle ne diminue. Les malades mentaux vivent un rejet social plus fort que ceux qui ont sombré dans des crises psychiques en raison d'expériences de vie négatives. Ils nous semblent étrangers, incompréhensibles et incontrôlables dans leurs actions, et à eux-mêmes aussi. Les diagnostics devant exercer une fonction protectrice médico-humaniste face au surmenage et au rejet ont ainsi l'effet inverse.

La psychiatrie s'est laissée manœuvrer dans une situation caractérisée par une grande incertitude et instabilité. Le projet Neuro-biologisation de la psychiatrie n'a jusqu'à présent pas pu apporter de résultats d'une évidence solide : Il n'existe pas de diagnostic, de génétique, de mécanismes originels biologiques, de pharmacothérapie à action causale, d'effets suffisants de la pharmacothérapie, de réduction de la tendance de chronicisation, d'interventions anticipées pharmacothérapeutiques efficaces. Il est impos- 
sible de déduire des syndromes valables déterminés par des processus spécifiques neurobiologiques uniquement à partir de symptômes. D'autre part, aucune classification de diagnostics cohérente ne peut actuellement être déduite des constats neurobiologiques. Cette faille doit être comblée par de nouveaux critères basaux de diagnostic. Les diagnostics cliniques doivent en revanche servir en première ligne à la compréhension et à la communication. Dans la mesure ils sont en principe le résultat d'un processus social de construction, une co-construction par le dialogue avec les autres significatifs est au cœur de l'univers social de la personne concernée. Dans ce contexte, une polyphonie interne et externe de ces constructions de la réalité est nécessaire et incontournable. La connaissance psychothérapeutique des experts est un élément de cette polyphonie.

\section{L'auteur}

Volkmar Aderhold, Dr. Med. est médecin en psychiatrie, psychothérapie et médecine psychothérapeutique ainsi qu'enseignant de la thérapie et du conseil systémiques (DGSF). Depuis 1982, il travaille dans la psychiatrie, dont 10 ans en tant que médecin-chef à la clinique de la psychiatrie et de la psychothérapie de la clinique universitaire Hambourg Eppendorf. Il collabore actuellement à l'Institut de la psychiatrie sociale à l'université de Greifswald et est actif par des présentations, publications et conseils d'organisations psychiatriques dans le secteur du développement structurel.

\section{Contact}

v.aderhold@gmx.de 